Bull. Austral. Math. Soc.

Vol. $71(2005)$ [417-424]

\title{
SEARCHING FOR SIMULTANEOUS ARITHMETIC PROGRESSIONS ON ELLIPTIC CURVES
}

\author{
Irene García-Selfa ANd José M. Tornero
}

We look for elliptic curves featuring rational points whose coordinates form two arithmetic progressions, one for each coordinate. A constructive method for creating such curves is shown, for lengths up to 5 .

\section{INTRODUCTION}

Let us consider an elliptic curve $E$ defined over $\mathbf{Q}$ by a general Weierstrass equation

$$
Y^{2}+a_{1} X Y+a_{3} Y=X^{3}+a_{2} X^{2}+a_{4} X+a_{6}, a_{i} \in \mathbf{Q}
$$

Definition: We shall say that the points $P_{0}, \ldots, P_{n} \in E$ are in (or form an) $x$-arithmetic progression if their $x$-coordinates are. The symmetric concept of $y$-arithmetic progression is defined analogously.

We denote by $S_{x}(E)$ and $S_{y}(E)$ the maximal number of points in $x$-arithmetic progression and $y$-arithmetic progression respectively that can be found in $E$.

REMARK. What we don't know about arithmetic progressions on elliptic curves is far more than what we know. Apparently the first one to consider the problem was Mohanty ([5]) who focused on the Mordell equation $Y^{2}=X^{3}+k$ and looked for integral points forming arithmetic progressions of difference 1 . He proved that for all these curves and for just these progressions $S_{x}(E) \leqslant 2$ and $S_{y}(E) \leqslant 4$.

Later on, Lee and Vélez [4] fixed their attention in the same family, but they took into consideration all possible progressions. They found infinite families of such curves satisfying $S_{x}(E) \geqslant 4$ and (not simultaneously) $S_{y}(E) \geqslant 6$.

Bremner, Silverman and Tzanakis [2] took another quite popular family of curves, $Y^{2}=X\left(X^{2}-n^{2}\right)$, and proved that for all these curves, and considering only integral points, $S_{x}(E) \leqslant 5$. They went further and proved very interesting results on this line concerning free subgroups of rank one in arbitrary elliptic curves. Their proofs were quite lengthy, involving very delicate computations of local heights.

Received 6th December, 2004

Both authors supported by FQM 218 and BFM 2001-3207 and FEDER.

Copyright Clearance Centre, Inc. Serial-fee code: 0004-9727/05 \$A2.00+0.00. 
After this (although it was published earlier) Bremner [1] carried out very clever computations in order to show that there are infinitely many curves satisfying $S_{x}(E) \geqslant 8$. Campbell [3] followed this line to produce curves with eight points in $x$-arithmetic progression and also a genus 1 curve with 12 such points (unfortunately the curve was not in Weierstrass form!). Maybe the more intriguing parts of Bremner's results are, on one side, the numerical evidence of the fact that the length of $x$-arithmetic progressions on elliptic curves may well not be bounded (although Bremner himself did not risk stating such a conjecture) and, on the other hand, the apparent connection between long arithmetic progressions and high ranks of the Mordell-Weil group.

From these last papers it becomes clear that the main problem, when one deals with arithmetic progressions on elliptic curves, is that the number of parameters involved (if one wants to work in full generality) becomes unmanageable. This is why the only precise results known are confined to one-parametric families.

This paper is devoted to finding simultaneous arithmetic progressions on elliptic curves. The precise definition goes as follows:

Definition: We shall say that the points $P_{0}, \ldots, P_{n} \in E$ are in (or form a) simultaneous arithmetic progression if their $x$-coordinates and their $y$-coordinates are arithmetic progressions (maybe not in the same order).

We shall denote by $S_{x, y}(E)$ the maximal number of points in simultaneous arithmetic progressions that can be found in $E$.

REMARK. When one looks for such progressions with more than three points, things start to become difficult, as the ordering of the points in both progressions may not coincide (see below for a precise explanation of this). Our initial aim, following [1], was to impose the conditions with full generality in order to narrow the search with respect to the $x$ arithmetic progression problem. Unfortunately it eventually became too complicated to deal with as well. So, we took a different point of view from Bremner [1]. We have worked with (almost) arbitrary elliptic curves, but we have looked for a restricted class of arithmetic progressions. With this starting point, we have been able to prove the following new results.

ThEOREM 1. There are elliptic curves over $\mathrm{Q}$ with $S_{y}(E) \geqslant 7$.

THEOREM 2. There are elliptic curves over $\mathbf{Q}$ with $S_{x, y}(E) \geqslant 5$.

\section{A CONSTRUCTION SCHEME}

As is well-known $([7])$, any change of variables preserving the Weierstrass form of $E$ must be of the form

$$
X^{\prime}=u^{2} X+r, \quad Y^{\prime}=u^{3} Y+s X+t
$$

so the existence (and the length) of $x$-arithmetic progressions is not affected by changes of variables. This also implies that, up to such a change, we can consider all the points in 
a certain $x$-arithmetic progression to be integral (the $y$ case being symmetric). Therefore as an immediate corollary of Siegel's theorem (see [6] for the original proof or [7] for a modern one) $E$ cannot contain infinite $x$-arithmetic progressions.

Hence we are left to study if there is a universal bound, independent of the chosen curve, for the length of arithmetic progressions. So, assume we have a curve with an $x$-arithmetic progression on it, say $P_{0}, \ldots, P_{n}$, and assume $[2] P_{0} \neq 0$. Then we can take $P_{0}$ to be $(0,0)$ and rotate the axes in order to take the tangent at $(0,0)$ to the line $X=0$ (warning: this may dismantle $y$-arithmetic progressions in the original model). Then our curve must look like

$$
E: Y^{2}+a X Y+b Y=X^{3}+c X^{2} .
$$

Now, if we make the change

$$
X \longmapsto\left(\frac{-c}{b}\right)^{3} X, Y \longmapsto\left(\frac{-c}{b}\right)^{2} Y,
$$

our curve will be defined by

$$
E(a, b): Y^{2}+a X Y+b Y=X^{3}-b X^{2} .
$$

This equation (also called Tate normal form) features two another obvious points in $E(a, b)$ other than $(0,0):(b, 0)$ and $(0,-b)$. Hence, in what follows we shall make a new (strong indeed) assumption and suppose $P_{1}=(b, 0)$. This implies that the difference in our $x$-arithmetic progression must be precisely $b$. In fact, we shall actually take $P_{0}=(0,-b)$ in order to avoid the repetition of 0 in the $y$-progression.

We must look for conditions which assure us that points $\left(k b, y_{k}\right)$ appear in $E(a, b)$. Furthermore, we want these points to form as well a $y$-arithmetic progression. Although the subindex of the points will represent the increasing order in the first coordinate it is obvious that, as far as the second coordinate is concerned, the subindex of a point might have nothing to do with its position in the $y$-arithmetic progression.

In order to do that, remember that if $P_{k}=\left(k b, y_{k}\right) \in E(a, b)$ then we must have

$$
y_{k}=\frac{-b(a k+1) \pm b \sqrt{(a k+1)^{2}+4 k^{2} b(k-1)}}{2} ;
$$

so proving the existence of $P_{k}$ is equivalent to finding a rational solution for the diophantine equation

$$
Z_{k}^{2}=(a k+1)^{2}+4 k^{2}(k-1) b .
$$

We now make the change of variables

$$
\alpha_{k}=a k+1+Z_{k}, \quad \beta_{k}=a k+1-Z_{k},
$$

and so our previous equation becomes

$$
\alpha_{k} \beta_{k}+4 k^{2}(k-1) b=0
$$


Now, when we gather together the equations for $P_{2}, \ldots, P_{n}$ we must take into account that, for all $k, \alpha_{k}+\beta_{k}=2 a k+2$. The diophantine system which is equivalent to the existence of our $x$-arithmetic progression is, therefore

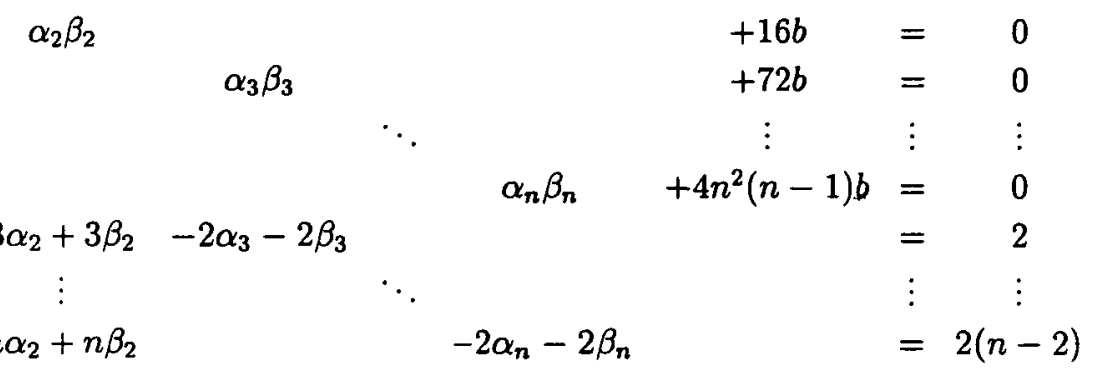

This can be viewed as the intersection of $(n-1)$ hyperquadrics and $(n-2)$ hyperplanes in the affine $(2 n-1)$-dimensional space over the rationals. Such a system is clearly unmanageable for, say $n=10$ (not to say less). Observe that, as $b=0$ leads to no progression at all, we must in fact ask all $\alpha_{i}$ and $\beta_{i}$ to be non-zero.

What looks particularly useful with this formulation of the problem is that, in this context, we can write

$$
y_{k}=\frac{b}{2}\left(-(a k+1) \pm Z_{k}\right)
$$

and hence the two solutions for $y_{k}$ are precisely, $-b \alpha_{k} / 2$ and $-b \beta_{k} / 2$. We can choose freely one of them, as the above equations are symmetric in $\left\{\alpha_{i}, \beta_{i}\right\}$. This is quite useful in order to force the existence of a simultaneous $y$-arithmetic progression.

\section{NumERICAL RESUlts}

Unlike the case of $x$-arithmetic progressions (for instance, in [1]), where the conditions for the existence of a progression of length, say, $k$ are also to be filled for the existence of a longer progression, in our case, if a set $P_{0}, \ldots, P_{n}$ displays a simultaneous arithmetic progression, that does not mean, in principle, that $P_{0}, \ldots, P_{k}$ also does (although we have not been able so far to find an example of this).

All the calculations in this section have been carried out with MapleV and PARI/GP. LENGTH 3. There are infinitely many curves with a simultaneous arithmetic progression of length 3. In fact, we may even ask both coordinates to share the same order in the progression. This clearly implies the three points must be collinear, and it also explains why one cannot hope to have such examples with longer lengths.

For instance, all curves of the family

$$
E(b): Y^{2}+(2 b-1) X Y+b Y=X^{3}-b X^{2}
$$

have the progression:

$$
\{(0,-b),(b, 0),(2 b, b)\}
$$


LENGTH 4. The system needed for the existence of an $x$-progression of length 4 is

$$
\begin{array}{ccc}
\alpha_{2} \beta_{2} & +16 b & =0 \\
\alpha_{3} \beta_{3}+72 b & =0 \\
3\left(\alpha_{2}+\beta_{2}\right)-2\left(\alpha_{3}+\beta_{3}\right) & & =2
\end{array}
$$

with $y_{2}=-\beta_{2} b / 2, y_{3}=-\beta_{3} b / 2$. Now, we can choose two values for $\beta_{2}$ and $\beta_{3}$ which will guarantee the existence of the $y$-arithmetic progression. These values, when replaced in the system will lead to a system of three linear equations in $\alpha_{2}, \alpha_{3}, b$ whose matrix is

$$
\left(\begin{array}{ccc|c}
\beta_{2} & 0 & 16 & 0 \\
0 & \beta_{3} & 72 & 0 \\
3 & -2 & 0 & 2-3 \beta_{2}+2 \beta_{3}
\end{array}\right)
$$

Hence we shall have a unique solution if $\beta_{2} \neq \beta_{3} / 3$, a family of solutions if $\beta_{2}=-2 / 3, \beta_{3}=-2$ and no solutions at all otherwise. The pair $\left(\beta_{2}=-2 / 3, \beta_{3}=-2\right)$ does not guarantee a length $4 y$-progression but it will appear later, in the length 5 study.

If we want 0 and $-b$ to be in the $y$-progression, there are only six possibilities for this sequence and they are precisely

$$
\begin{aligned}
\{(2 b, b, 0,-b),(b, 0,-b,-2 b),(0,-b,-2 b,-3 b), & \left(\frac{b}{2}, 0, \frac{-b}{2},-b\right) \\
& \left.\left(0, \frac{-b}{2},-b, \frac{-3 b}{2}\right),\left(0, \frac{-b}{3}, \frac{-2 b}{3},-b\right)\right\} .
\end{aligned}
$$

Each of them allows two possible choices for $\beta_{2}$ and $\beta_{3}$, except the penultimate case, in which $\left(\beta_{2}=1, \beta_{3}=3\right)$ is forbidden. The results found are shown in the following table, except the cases $\left(\beta_{2}=-2, \beta_{3}=-4\right)$, which leads to a degenerate case $b=0$, and $\left(\beta_{2}=4, \beta_{3}=6\right)$, which gives the same curve as $\left(\beta_{2}=4, \beta_{3}=-2\right)$.

\begin{tabular}{cccc}
$\left(\beta_{1}, \beta_{2}\right)$ & $(a, b)$ & $S_{x}$ & $S_{y}$ \\
\hline$(-4,-2)$ & $(-5 / 3,-1 / 6)$ & $\geqslant 5$ & $\geqslant 5$ \\
$(-2,4)$ & $(-7 / 15,4 / 15)$ & $\geqslant 5$ & $\geqslant 4$ \\
$(-1,1)$ & $(-29 / 48,7 / 192)$ & $\geqslant 4$ & $\geqslant 4$ \\
$(2 / 3,4 / 2)$ & $(-7 / 9,2 / 27)$ & $\geqslant 4$ & $\geqslant 5$ \\
$(1,-1)$ & $(-5 / 16,1 / 64)$ & $\geqslant 6$ & $\geqslant 7$ \\
$(4 / 3,2 / 3)$ & $(-7 / 45,-1 / 270)$ & $\geqslant 4$ & $\geqslant 4$ \\
$(3,1)$ & $(29 / 96,-5 / 128)$ & $\geqslant 4$ & $\geqslant 4$ \\
$(4,-2)$ & $(1 / 3,1 / 6)$ & $\geqslant 4$ & $\geqslant 5$ \\
$(6,4)$ & $(25 / 21,-2 / 7)$ & $\geqslant 6$ & $\geqslant 4$
\end{tabular}

This search gave us the first interesting example, announced in Theorem 1: the existence of an elliptic curve with a $y$-arithmetic progression of length 7 , a fact not reported until now, as far as we know. We shall look more closely at this example below. 
LENGTH 5 . The system needed for the $x$-progression is

$$
\begin{array}{ccccc}
\alpha_{2} \beta_{2} & & +16 b & =0 \\
& \alpha_{3} \beta_{3} & & +72 b & =0 \\
& & \alpha_{4} \beta_{4}+192 b & =0 \\
3\left(\alpha_{2}+\beta_{2}\right) & -2\left(\alpha_{3}+\beta_{3}\right) & & & =2 \\
4\left(\alpha_{2}+\beta_{2}\right) & & -2\left(\alpha_{4}+\beta_{4}\right) & & =4
\end{array}
$$

where $y_{j}=-\beta_{j} b / 2$, for $j=2,3,4$. Observe now that a blind choice of $\left(\beta_{2}, \beta_{3}, \beta_{4}\right)$ as in the previous case will lead, in general, to an incompatible system of equations.

In fact, the rank of the coefficient matrix is 4 , except in the case $\beta_{2}=\beta_{3} / 3=\beta_{4} / 6$ but, in this case the system is incompatible. However, these relations will prove useful later on.

There are ten possible progressions of length 5 containing both 0 and $-b$, each of them permitting six different choices for the triple $\left(\beta_{2}, \beta_{3}, \beta_{4}\right)$, which are the permutations of a single choice. From this 60 cases only two led to a compatible system. This two cases, shown below, prove therefore Theorem 2 .

CASE 1. $\beta_{2}=-4, \beta_{3}=-2, \beta_{4}=-6$

This triple gives the curve $E(-5 / 3,-1 / 6)$ with the following points lying on it:

$$
\left\{\left(0, \frac{1}{6}\right),\left(\frac{-1}{6}, 0\right),\left(\frac{-2}{6}, \frac{-2}{6}\right),\left(\frac{-3}{6}, \frac{-1}{6}\right),\left(\frac{-4}{6}, \frac{-3}{6}\right)\right\},
$$

which form a simultaneous arithmetic progression of length 5 .

CASE 2. $\beta_{2}=1, \beta_{3}=-1, \beta_{4}=-2$

The resulting curve is $E(-5 / 16,1 / 64)$ with the following points:

$$
\left\{\left(0, \frac{-2}{128}\right),\left(\frac{1}{64}, 0\right),\left(\frac{2}{64}, \frac{-1}{128}\right),\left(\frac{3}{64}, \frac{1}{128}\right),\left(\frac{4}{64}, \frac{2}{128}\right)\right\}
$$

which form a simultaneous arithmetic progression (note that this curve also appeared in the previous case). Furthermore, other points lying on the curve are

$$
\left(\frac{1}{8}, \frac{-4}{128}\right)\left(\frac{-1}{32}, \frac{-3}{128}\right),\left(\frac{5}{64}, \frac{-1}{64}\right)
$$

hence as noted above $S_{x} \geqslant 6, S_{y} \geqslant 7$, although there are no simultaneous progressions in the curve of length 6 .

REMARK. As we mentioned at the beginning of the section, both examples of simultaneous arithmetic progressions of length 5 also have arithmetic progressions of length 4 . This is also the case with the nine examples of length 4 found. However, this does not seem to us to be enough to conjecture that this holds in general. 
Back to our search. Another reasonable way of constructing simultaneous arithmetic progressions of length 5 seems to be using 0 and $-b(a+1)$ as terms of our progression and again we have 60 possibilities, but none lead to a solution, as we now show.

CASE 1. $\beta_{2}=4(a+1), \beta_{3}=6(a+1), \beta_{4}=8(a+1)$

If this choice made sense, we would have the $y$-progression

$$
\{0,-b(a+1),-2 b(a+1),-3 b(a+1),-4 b(a+1)\} .
$$

As we said above, there are 5 more possibilities giving this sequence, permuting the values of $\beta_{2}, \beta_{3}$ and $\beta_{4}$, but this one suits us well as an illustration. Our system matrix is now

$$
\left(\begin{array}{ccccc}
-4(a+1) & 0 & 0 & 16 & 0 \\
0 & -6(a+1) & 0 & 72 & 0 \\
0 & 0 & -8(a+1) & 192 & 0 \\
3 & -2 & 0 & 0 & 2 \\
4 & 0 & -2 & 0 & 4
\end{array}\right)
$$

with determinant $-3072(a+1)^{2}$. Hence we must take $a=-1$ if we want the system to have solution, but this case is degenerate.

CASE 2. $\beta_{2}=6(a+1), \beta_{3}=4(a+1), \beta_{4}=8(a+1)$

This case is also impossible, but for different reasons: it has a matrix with determinant $7168(a+1)^{2}(4 a+5)$. The choice $a=-5 / 4$ leads to a compatible system. But remember that it also must satisfy

$$
a=\frac{\alpha_{i}+\beta_{i}-2}{2 i}, i=2,3,4,
$$

which is not true in this case. In fact, this extra condition annihilates the advantage of making such a choice, because the value of the parameter cannot be truly arbitrary.

\section{Final REMARKS}

The above arguments, when applied to a length $n$ simultaneous arithmetic progression lead to $n(n-1) / 2$ progressions with $(n-2)$ ! possible value choices for each one. That is, $n ! / 2$ systems have to be checked. We have done the 360 calculations for $n=6$, obtaining no simultaneous progressions. Of course, that does not mean that the case $n=7$ will also be unsuccessful, as we noted before, but most probably this kind of search will not produce further results.

As a final comment, we would like to stress possible ways for expanding the results in this paper:

(a) The first obvious thing to do it is enlarging the scope of the progressions considered. Full generality, although desirable, may be too messy to deal with, at least with the 
current state of the art. A simpler way could be, for instance, considering $x$-progressions in which $b$ appears, although not necessarily as the first term. However, this has also proved to be unsuccessful for $n=6$ (at a cost of around 72 CPU hours).

(b) While looking for $y$-progressions seems difficult, the formulation used here for the existence of $x$-progressions of given length can be found useful. In a future paper, we expect to explore this presentation of the problem, with the help of elimination theory and Gröbner bases.

\section{REFERENCES}

[1] A. Bremner, 'On arithmetic progressions on elliptic curves', Experiment. Math. 8 (1999), 409-413.

[2] A. Bremner, J.H. Silverman and N. Tzanakis, 'Integral points in arithmetic progression on $y^{2}=x\left(x^{2}-n^{2}\right)$ ', J. Number Theory 80 (2000), 187-208.

[3] G. Campbell, 'A note on arithmetic progressions on elliptic curves', J. Integer Seq. 6 (2003), 03.1.3.

[4] J.-B. Lee and W.Y. Vélez, 'Integral solutions in arithmetic progression for $y^{2}=x^{3}+k$ ', Period. Math. Hungar. 25 (1992), 31-49.

[5] S.P. Mohanty, 'On consecutive integral solutions for $y^{2}=x^{3}+k$ ', Proc. Amer. Math. Soc. 48 (1975), 261-265.

[6] C.L. Siegel, 'Über einige Anwendungen diophantischer Approximationen (1929)', in Collected works (Springer-Verlag, Berlin, Heidelberg, New York, 1966), pp. 209-266.

[7] J. Silverman, The arithmetic of elliptic curves (Springer-Verlag, Berlin, Heidelberg, New York, 1986).

Deptartamento de Álgebra

Facultad de Matemáticas

Universidad de Sevilla

Apdo. 1160

41080 Sevilla

Spain

e-mail: igselfa@us.es

tornero@algebra.us.es 